\title{
Functional 2D Procrustes Shape Analysis
}

\author{
Rasmus Larsen \\ Informatics and Mathematical Modelling, Technical University of Denmark \\ Richard Petersens Plads, Building 321, DK-2800 Kgs. Lyngby, Denmark \\ rl@imm.dtu.dk, http://www.imm.dtu.dk/image
}

\begin{abstract}
Using a landmark based approach to Procrustes alignment neglects the functional nature of outlines and surfaces. In order to reintroduce this functional nature into the analysis we will consider alignment of shapes with functional representations. First functional Procrustes analysis of curve shapes is treated. Following this we will address the analysis of surface shapes.
\end{abstract}

\section{Introduction}

In this paper we consider the representation and alignment of two dimensional points sets, curves and surfaces. The curves and surfaces may arise as outlines and areas delineated by outlines of two dimensional objects or cross sections or projections of three dimensional objects. Intuitively and formalized in the definition by [1] an object's shape is invariant under a Euclidean similarity transformation. Often a set of curves delineates an area of interest. In some of these cases it may then be appropriate to consider alignment with respect to the interior of these objects instead of their outline or landmarks on their outline. Prior to any modelling of shape we need to filter out these nuissance parameters of a Euclidean similarity transformation from each object in our data set. We define functional Procrustes analysis based on spline based representations of outlines as well as spline based representations of regions. Thus generalizing the method of generalized Procrustes analysis (GPA) based on sets of labelled landmarks [2, 3, 4].

\section{Functional Generalized Procrustes Analysis}

In the following two representations of shapes are considered, namely functional curve and surface representations. A functional 2D curve shape consisting of an open or closed continuous curve is represented by a continuous complex function $y(s):[0, l] \rightarrow \mathbb{C}$. For closed curves $y(0)=y(l)$. In our applications we consider all such curves with the exception of curves for which $y(s)=$ const. for all $s \in[0, l]$. The centroid of a functional curve shape is given by $\bar{y}=\frac{1}{l} \int_{0}^{l} y(s) d s$ A curve with centroid 0 is said to be centered. The centroid size is $S(y)=$ 
$\left\{\int_{0}^{l}|y(s)-\bar{y}|^{2} d s\right\}^{1 / 2}$ We may choose to use the natural parameterization of the curves. However, generally the correspondences between outlines of biological and other objects are not given by the normalized distance traversed along the outline. More often we assume that the correspondence between such outlines are given at sets of manually or (semi)-automatically identified anatomical and/or geometrical landmarks along the outlines, and natural or other parameterizations are used to define correspondences between landmarks. In other situations the correspondences have to be estimated directly from data.

A functional 2D surface shape consisting of a surface patch is represented by a continuous complex function $y(s): \Omega \rightarrow \mathbb{C}$. In our application we will consider such surface patches that are constrained to have non-zero area. The centroid of a functional surface shape is given by $\bar{y}=\frac{1}{|\Omega|} \int_{\Omega} y(s) d s$, where $|\Omega|$ is the size(area) of the parameter space. A surface with centroid 0 is said to be centered. The centroid size is $S(y)=\left\{\int_{\Omega}|y(s)-\bar{y}|^{2} d s\right\}^{1 / 2}$

\subsection{Functional Curve Shapes}

Let us consider two curves $y(s), w(s):[0, l] \rightarrow \mathbb{C}$. Without loss of generality we assume that the curves have been centered, i.e. $\int_{0}^{l} y(s) d s=\int_{0}^{l} w(s) d s=0$.

Definition 1. The full functional Procrustes fit of $w$ onto $y$ is $w^{P}(s)=\hat{a}+\hat{b} w(s)$ where $(\hat{a}, \hat{b})$ is chosen to minimize

$$
D^{2}(y, w)=\int_{0}^{l}|y(s)-b w(s)-a|^{2} d s .
$$

$a \in \mathbb{C}$ is a translation vector, $\beta=\bmod (b) \in \mathbb{R}_{+}$is a scale parameter, and $0 \leq \theta=\arg (b)<2 \pi$ is a rotation .

Result 1. The full functional Procrustes fit has matching parameters

$$
\hat{a}=0 \quad \hat{b}=\frac{\int_{0}^{l} w(s)^{*} y(s) d s}{\int_{0}^{l} w(s)^{*} w(s) d s}
$$

Proof. Omitted, follows from differentiation of the objective function.

To obtain a symmetric measure of shape distance we standardize the curve shapes to unit size. The objective function then becomes

Definition 2. The full functional curve shape Procrustes distance between shapes $y$ and $w$ is given by

$$
d_{F}^{C}(y, w)=\left\{1-\frac{\int_{0}^{l} y(s)^{*} w(s) d s \int_{0}^{l} w(s)^{*} y(s) d s}{\int_{0}^{l} y(s)^{*} y(s) d s \int_{0}^{l} w(s)^{*} w(s) d s}\right\}^{1 / 2}
$$




\subsection{Functional Procrustes Mean Curve Shape}

Let a sample of $n$ two dimensional curves given by $w_{i}(s):[0, l] \rightarrow(C)$ be available from the pertubation model

$$
w_{i}(s)=a_{i}+b_{i}\left(\mu(s)+\epsilon_{i}(s)\right), \quad i=1, \ldots, n,
$$

where $a_{i} \in \mathbb{C}$ are translation vectors, $\beta_{i}=\bmod \left(b_{i}\right) \in \mathbb{R}_{+}$are scale parameters, $0 \leq \theta_{i}=\arg \left(b_{i}\right)<2 \pi$ are rotations, $\epsilon_{i}(s):[0, l] \in \mathbb{C}$ are independent zero mean complex random error functions, and $\mu$ is the population mean curve. Under this model it is possible to estimate the shape of $\mu,[\mu]$.

Definition 3. The functional Procrustes estimate of mean shape $[\hat{\mu}]$ is obtained by minimization over $\mu$ of the sum of square functional Procrustes distances from each $w_{i}$ to a fixed size mean configuration $\mu$, i.e.

$$
[\hat{\mu}]=\arg \inf _{\mu} \sum_{i=1}^{n}\left(d_{F}^{C}\right)^{2}\left(w_{i}, \mu\right)
$$

\subsection{Functional Curve Shape Representation}

A convenient representation of curves is based on linear basis expansions in $s$

$$
y(s)=\sum_{m=1}^{M} c_{m} h_{m}(s), \quad s \in[0, l],
$$

where $c_{m} \in \mathbb{C}, h(s):[0, l] \rightarrow \mathbb{R}$. For closed curves $y(0)=y(l)$. The parameter $s$ provides the correspondence between curves. A centered curve shape is obtained by translating the linear basis function coefficients by $\boldsymbol{w}^{T} \boldsymbol{c} / \boldsymbol{w}^{T} \mathbf{1}_{M}$, where $\boldsymbol{w}=$ $\int_{0}^{l} \boldsymbol{h}(s) d s$. Let two centered curves be given by linear combinations of the same set of basis functions, i.e.

$$
y(s)=\sum_{m=1}^{M} c_{m} h_{m}(s)=\boldsymbol{h}(s)^{T} \boldsymbol{c}, \quad w(s)=\sum_{m=1}^{M} d_{m} h_{m}(s)=\boldsymbol{h}(s)^{T} \boldsymbol{d},
$$

where we have introduced a vector notation for the coefficients and basis functions: $\boldsymbol{c}=\left(c_{1}, \ldots, c_{M}\right)^{T}, \boldsymbol{d}=\left(d_{1}, \ldots, d_{M}\right)^{T}$, and $\boldsymbol{h}(s)=\left(h_{1}(s), \ldots, h_{M}(s)\right)^{T}$.

Result 2. For the full functional Procrustes fit of the curve $w$ onto the curve $y$ using the same set of basis functions $\boldsymbol{h}(s)$ the matching parameters are

$$
\hat{a}=0 \quad \hat{b}=\frac{\boldsymbol{d}^{*} \boldsymbol{A c}}{\boldsymbol{d}^{*} \boldsymbol{A d}}=\frac{(\boldsymbol{L d})^{*}(\boldsymbol{L} \boldsymbol{c})}{(\boldsymbol{L d})^{*}(\boldsymbol{L d})}
$$

where $\boldsymbol{A}=\boldsymbol{L} \boldsymbol{L}^{T}=\int_{0}^{l} \boldsymbol{h}(s) \boldsymbol{h}(s)^{T} d s$ is a positive definite matrix, and $\boldsymbol{L}$ is the lower triangular matrix resulting from a Cholesky decomposition of $\boldsymbol{A}$ 
Proof. Follows directly from Result 1

Let a sample of $n$ centered curves be given by

$$
w_{i}(s)=\sum_{m=1}^{M} d_{i m} h_{m}(s)=\boldsymbol{h}(s)^{T} \boldsymbol{d}_{i}, \quad i=1, \ldots, n, \quad \boldsymbol{d}_{i}=\left(d_{i 1}, \ldots, d_{i M}\right)^{T}
$$

Result 3. The coefficients of the full functional Procrustes estimate of the mean curve of the sample of curves $w_{i}$ are then $\boldsymbol{e}=\boldsymbol{L}^{-1} \boldsymbol{v}$, where $\boldsymbol{v}$ is the eigenvector corresponding to the largest eigenvalue of the Hermitian complex sum of squares and products matrix

$$
\boldsymbol{C}=\sum_{i=1}^{n} \frac{\left(\boldsymbol{L} \boldsymbol{d}_{i}\right)\left(\boldsymbol{L} \boldsymbol{d}_{i}\right)^{*}}{\left(\boldsymbol{L} \boldsymbol{d}_{i}\right)^{*}\left(\boldsymbol{L} \boldsymbol{d}_{i}\right)}
$$

Proof. Obviously, the minimizing $\mu(s)$ must belong to the same linear subspace as the sample of curves. Subject to $S(\mu)=1$ we seek the minimizer of

$$
\begin{aligned}
\sum_{i=1}^{n} d_{F}^{2}\left(\mu, w_{i}\right) & =\sum_{i=1}^{n}\left\{1-\frac{\int_{0}^{l} \mu(s)^{*} w_{i}(s) d s \int_{0}^{l} w_{i}(s)^{*} m u(s) d s}{\int_{0}^{l} \mu(s)^{*} \mu(s) d s \int_{0}^{l} w_{i}(s)^{*} w_{i}(s) d s}\right\} \\
& =n-\sum_{i=1}^{n}\left\{\frac{\boldsymbol{e}^{*} \boldsymbol{A} \boldsymbol{d}_{i} \boldsymbol{d}_{i}^{*} \boldsymbol{A} \boldsymbol{e}}{\boldsymbol{e}^{*} \boldsymbol{A} \boldsymbol{e} \boldsymbol{d}_{i}^{*} \boldsymbol{A} \boldsymbol{d}_{i}^{*}}\right\}=n-\frac{\boldsymbol{v}^{*} \boldsymbol{C} \boldsymbol{v}}{\boldsymbol{v}^{*} \boldsymbol{v}}
\end{aligned}
$$

where $\boldsymbol{A}=\boldsymbol{L} \boldsymbol{L}^{T}=\int_{0}^{l} \boldsymbol{h}(s) \boldsymbol{h}(s)^{T} d s$ is a positive definite matrix, $\boldsymbol{L}$ is the lower triangular matrix resulting from a Cholesky decomposition of $\boldsymbol{A}$, and $\boldsymbol{v}=\boldsymbol{L} \boldsymbol{e}$. Since

$$
S(\mu)=\sqrt{\int_{0}^{l} \mu(s)^{*} \mu(s) d s}=\sqrt{\boldsymbol{e}^{*} \int_{0}^{l} h(s) h(s)^{T} d s \boldsymbol{e}}=\sqrt{\boldsymbol{e}^{*} \boldsymbol{A} \boldsymbol{e}}=\|\boldsymbol{L} \boldsymbol{e}\|
$$

we have $\boldsymbol{L} \hat{\boldsymbol{e}}=\arg \sup _{\|v\|=1} \boldsymbol{v}^{*} \boldsymbol{C} \boldsymbol{v}$ and therefore $\hat{\boldsymbol{e}}=\boldsymbol{L}^{-1} \hat{\boldsymbol{v}}$

Hence, $\hat{\mu}$ is given by the coefficients $\hat{\boldsymbol{e}}$ obtained as the complex eigenvector corresponding to the largest eigenvalue of $\boldsymbol{C}$. Again rotations of $\hat{\boldsymbol{e}}$ also yield solutions, but all corresponding to the same curve shape.

\subsection{Functional Curve Shape Parameterization}

We have not yet discussed the choice of curve parameterization. In some situations the parameterization may be given by the nature of the data at hand. In other situations the natural parameterization of curve length is appropriate. In many situations the correspondence between curves are given at finite series of landmarks. These may be manually or automatically identified geometrical and anatomical landmarks. 
Let there be given $n$ closed curves, $y_{i}, i=1, \ldots, n$ with the constraints $y_{i}\left(\xi_{0}\right)=y_{i k}, y_{i}\left(\xi_{j}\right)=y_{i j}$ for $j=1, \ldots, k$, where $0=\xi_{0} \leq \xi_{1} \leq \cdots \leq \xi_{k}=l$. Let the curve lengths between landmarks and the total length of the $i$ th curve be

$$
l_{i j}=\int_{\xi_{j-1}}^{\xi_{j}}\left|y^{\prime}(s)\right| d s, \quad L_{i}=\int_{\xi_{0}}^{\xi_{k}}\left|y^{\prime}(s)\right| d s=\sum_{j=1}^{k} l_{i j},
$$

then a parameterization $s \in[0, l]$ based on normalized average curve length between landmarks is given by

$$
\xi_{0}=0, \quad \xi_{j}=\xi_{j-1}+\frac{l}{n} \sum_{i=1}^{n} l_{i j} / L_{i},
$$

This parameterization is based on normalizing the curve segments $l_{i j}$ with respect to the length of each curve. Instead of these normalized curve segment lengths we could use the average curve segment length from the Procrustes aligned curves, $l_{i j}^{P}$, i.e.

$$
\xi_{0}^{P}=0, \quad \xi_{j}^{P}=\xi_{j-1}^{P}+l \sum_{i=1}^{n} l_{i j}^{P} / \sum_{i=1}^{n} L_{i}^{P}=\xi_{j-1}^{P}+l l_{i j}^{\mu} / L_{i}^{\mu},
$$

$l_{i j}^{\mu}$ is the curve segment lengths for the Procrustes mean curve. However, because parameterization precedes Procrustes alignment this has to be done iteratively.

Let us consider at set of outlines of hands, 10 images each of 4 individuals were taken 1. 56 landmarks are chosen as is shown in Figure 1(a). We will compare Procrustes alignment based on landmark and functional approaches.

Given landmarks $\boldsymbol{y}=\left(y_{1}, \ldots, y_{k}\right)^{T}, k=56$, we employ a periodic linear spline, $y(s), \xi_{0} \leq s \leq \xi_{k}$ - i.e. a second order B-spline - to interpolate the points $\left(\xi_{0}, y_{k}\right),\left(\xi_{1}, y_{1}\right), \ldots,\left(\xi_{k}, y_{k}\right)$. The parameterization is based on the average curve segment length in the Procrustes aligned curves, and is determined iteratively.

Let $B_{i, 2}(s)$ be the $k+1$ second order B-spline basis functions corresponding to knots $\boldsymbol{\xi}$ (cf. [5]. Then we have

$$
h_{i}(s)=B_{2, i+1}(s) \quad i=1, \ldots, k-1 ; \quad h_{k}(s)=B_{2,1}(s)+B_{2, k+1}(s)
$$

Fitting the data $y\left(\xi_{0}\right)=y_{k}, y\left(\xi_{j}\right)=y_{j}$ for $j=1, \ldots, k$ yields a linear system of equations to which the solution trivially is $\hat{c}_{i}=y_{i}$, or in vector notation $\hat{\boldsymbol{c}}=\boldsymbol{y}$. Now $\boldsymbol{w}=\int_{\xi_{0}}^{\xi_{k}} \boldsymbol{h}(s) d s$ and $\boldsymbol{A}=\int_{\xi_{0}}^{\xi_{k}} \boldsymbol{h}(s) \boldsymbol{h}(s)^{T} d s$ can be determined. For arbitrary knot sequences $\xi=\left\{\xi_{0}, \ldots, \xi_{k}\right\}$ with knot spacing $d_{i}=\xi_{i}-\xi_{i-1}$.

$$
\boldsymbol{w}=\frac{1}{2}\left[\begin{array}{c}
d_{1}+d_{2} \\
d_{2}+d_{3} \\
\cdot \\
d_{k-1}+d_{k} \\
d_{k}+d_{1}
\end{array}\right] \quad \boldsymbol{A}=\frac{1}{6}\left[\begin{array}{cccc}
4\left(d_{1}+d_{2}\right) & d_{2} & & d_{1} \\
d_{2} & 4\left(d_{2}+d_{3}\right) & d_{3} & \\
& \cdot & \cdot & \cdot \\
\\
& & d_{k-1} 4\left(d_{k-1}+d_{k}\right) & d_{k} \\
d_{1} & & d_{k} & 4\left(d_{k}+d_{1}\right)
\end{array}\right]
$$

\footnotetext{
${ }^{1}$ The data are available from www.imm.dtu.dk/ aam
} 


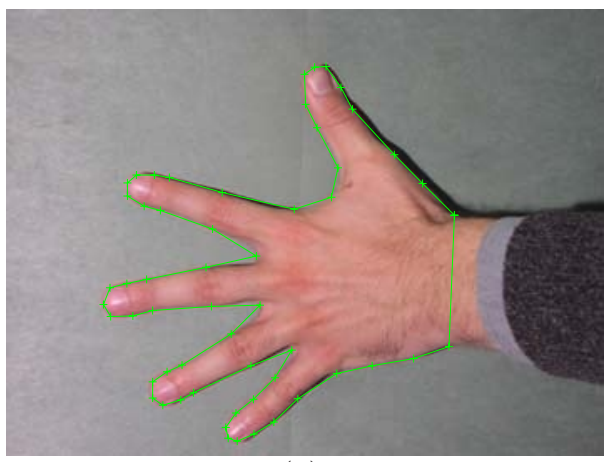

(a)

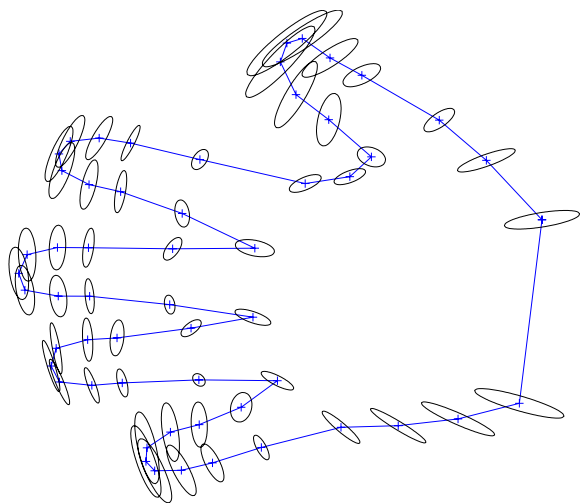

(b)

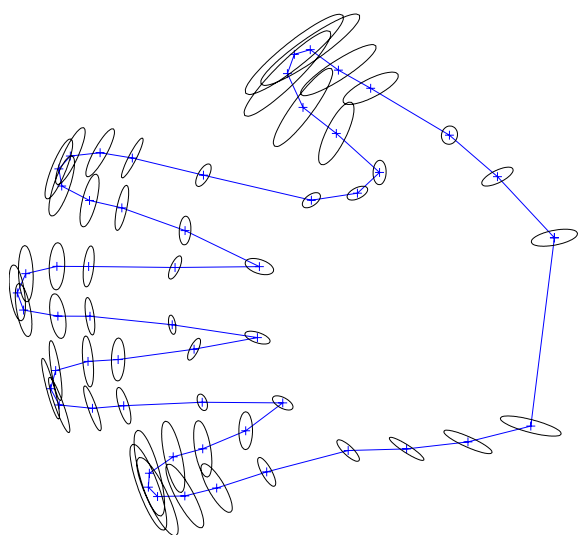

(c)

Fig. 1. (a) 56 landmarks on the outline of a hand. Landmark based (b) and functional curve (c) Generalized Procrustes Alignment of hand images. The scatter of the full Procrustes fits of the curves at each landmark is shown by a contour ellipse at 1 standard deviation

In Figure 1 the results of landmark based and functional generalized Procrustes analysis of the hand data at shown. Segments where landmarks are relatively more dispersed receive larger weight in the functional analysis, hence the scatter becomes relatively smaller than compared with the landmark based approach.

\subsection{Surface Generalized Procrustes Analysis}

For the alignment of surface patches, $y(s), w(s): \Omega \rightarrow \mathbb{C}$ similar results as for curve shapes exist. The only difference being that all integrals are over $\Omega$ instead of $[0, l]$. As for the curve shapes a crucial element is the parameterization of the surface shapes. Again we will discuss a parameterization derived from a finite series of landmarks. Let there be given $n$ surface shapes, $y_{i}, i=1, \ldots, n$ with 
landmarks $y_{i}\left(\xi_{j}\right)=y_{i j}$ for $j=1, \ldots, k$, where $\xi_{j} \in \Omega$ is the surface parameter for the $j$ th landmark. Obtaining a parameterization is closely related to defining a warp function between the shapes under consideration. A warp function is a spatial transformation from one spatial configuration into another. 6] give a survey of warping methods. We will consider the simplest construction of a warp by assuming a piece-wise affine function. We will base this function on a mesh constructed by a Delaunay triangulation.

We begin by choosing a reference shape. This reference shape may be the landmark based Procrustes mean shape estimated from the set of landmarks on all shapes. We approximate the outline of the surface area of the reference shape by linear splines of (some of) the landmarks. Having done this we can partition the surface area by the set of triangles of a Delaunay triangulation that reside inside the outline of the surface area. The Delaunay triangulation partitions the convex hull of the landmarks. However, we only retain those triangles inside the outline of the surface area. Knowing the order of vertices of the surface area outline it is easily determined by inspection of the triangle vertices order if a triangle is inside the surface area.

Now, a crucial assumption is that by applying the reference shape Delaunay triangulation to each of the $n$ shapes of the data set we will obtain a one-toone mapping. This will generally not be true. However, for shape sets with low variability this is not an unreasonable condition and it can easily be tested by examining triangle normals. Let $\Omega$ be the surface patch of the complex plane corresponding to the reference surface shape, and let the parameterization of the shape data set be given by affinely warping the Delaunay triangles of the reference shape to the corresponding triangles of each of the surface shapes.

This procedure is realized by choosing a representation in based on $M=k$ pyramidal basis functions. Each function is centered at a landmark where it has value 1 ; it is only non-zero in the Delaunay triangles in which that landmark is a vertex; it varies linearly within each triangle, and has value 0 at the 2 other vertices. In Figure 2(b) one basis function spanning in this case 4 Delaunay triangles is shown. The optimal coefficients in Equation (2) are trivially equal to the landmark coordinates. In order to determine the weight matrix, $\boldsymbol{A}$ we partition the surface shape, $\Omega$ into mutually exclusive patches given by the Delaunay triangles, $\Omega_{t}, t=1, \ldots, T$ i.e.

$$
\boldsymbol{A}=\int_{\Omega} \boldsymbol{h}(s) \boldsymbol{h}^{T}(s) d s=\sum_{i=1}^{T} \int_{\Omega_{i}} \boldsymbol{h}(s) \boldsymbol{h}^{T}(s) d s
$$

Let $\phi_{i}: B \rightarrow \mathbb{C}$ be an affine function that maps $B: 0 \leq u \leq 1,0 \leq v \leq u$ to the $i$ 'th Delaunay triangle. Then with this Delaunay triangle the basis functions that are non-zero here are equal to one of these element functions

$$
\left.\begin{array}{ll}
f_{1}\left(\phi_{i}(u, v)\right)= & u \\
f_{2}\left(\phi_{i}(u, v)\right)= & v \\
f_{3}\left(\phi_{i}(u, v)\right)=1 & -u-v
\end{array}\right\} \quad \text { for } 0 \leq u \leq 1,0 \leq v \leq u
$$




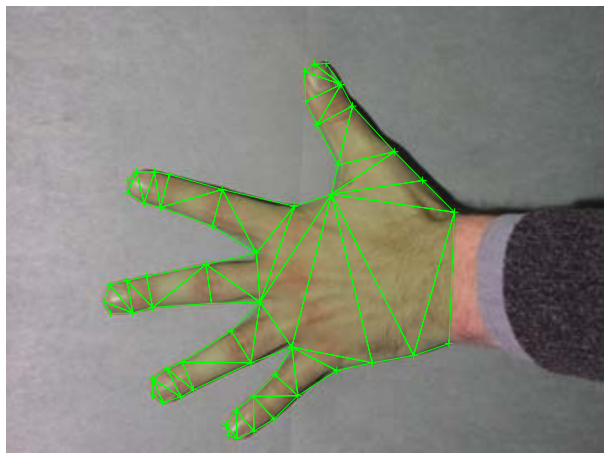

(a)

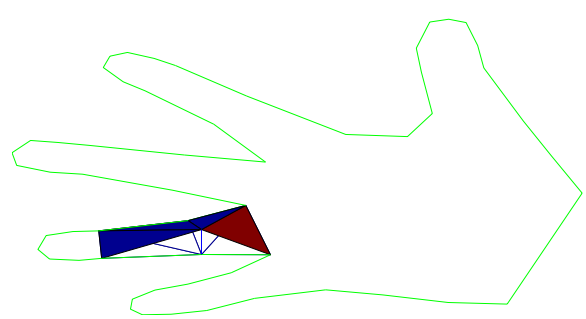

(b)

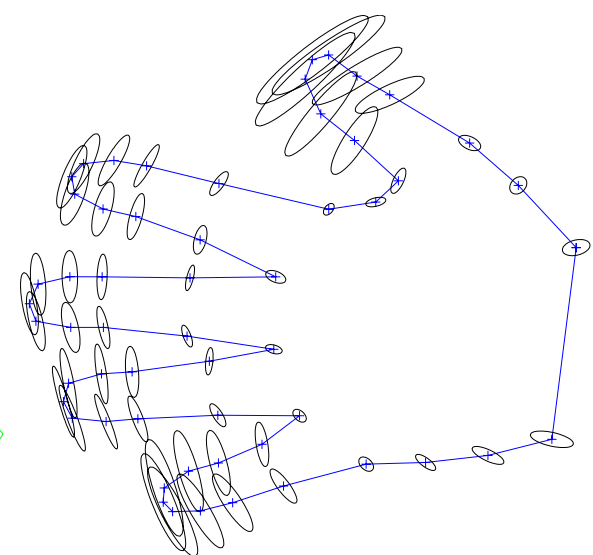

(c)

Fig. 2. (a) Annotated hand with Delaunay triangulation of landmarks; (c) $m$ th basis function; (c) Surface based functional Procrustes alignment of the hand images. he scatter of the full Procrustes fits of the curves at each landmark is shown by a contour ellipse at 1 standard deviation. Compare Figure 1

For those pairs of basis functions $(j, k)$ that are non-zero within the $i$ th Delaunay triangle, let $\tau_{i j}, \tau_{i k} \in\{1,2,3\}$ identify which elementar function the $j$ th and $k$ th basis functions consist of. Then we have

$$
\int_{\Omega_{i}} h_{j}(s) h_{k}(s) d s= \begin{cases}\operatorname{Ar}\left(\Omega_{i}\right) / 6 & \text { for } j=k \\ \operatorname{Ar}\left(\Omega_{i}\right) / 12 & \text { for } j \neq k\end{cases}
$$

Now within each Delaunay triangle 3 basis functions are non-zero. We can compute their contributions from Equation (3) and update $\boldsymbol{A}$ accordingly.

In Figure 2(a) based on the landmarks shown in Figure 1(a) the triangles of the Delaunay triangulation that belong to the interior of the hand are shown. The Delaunay triangulation is determined from a landmark based Procrustes mean of the hand data set. Following the procedure described above we arrive 
at the surface shape Procrustes alignment illustrated in Figure 2(c). Compared to the landmark based and curve shape based alignments shown in Figure 1 we obtain an even better alignment of the bulk of the hand. The major part of the variation is transferred to the fingers/fingertips.

\section{Conclusion}

We have derived procrustes methods for the alignment of curve and surface shapes based on functional representations using arbitrary parameterizations. In particular we have examined natural curve parameterizations. We have demonstrated that functional representations based on natural parameterizations and functional procrustes methods result in more intuitive alignment of sets of shapes.

\section{References}

1. Kendall, D.G.: The diffusion of shape. Advances in Applied Probability 9 (1977) 428-430

2. Gower, J.C.: Generalized Procrustes analysis. Psychometrika 40 (1975) 33-50

3. ten Berge, J.M.F.: Orthogonal Procrustes rotation for two or more matrices. Psychometrika 42 (1977) 267-276

4. Goodall, C.: Procrustes methods in the statistical analysis of shape. Journal of the Royal Statistical Society, Series B 53 (1991) 285-339

5. Nielsen, H.B.: Cubic splines (1998)

6. Glasbey, C.A., Mardia, K.V.: A review of image warping methods. Journal of Applied Statistics 25 (1998) 155-171 